\title{
Matching Semantic Web Services Using Different Ontologies
}

\author{
Le Duy Ngan and Angela Goh \\ School of Computer Engineering, Nanyang Technological University, Singapore \\ \{1edu0001,ASESGOH\}@ntu.edu.sg \\ http://www.ntu.edu.sg
}

\begin{abstract}
The rapid development of semantic web services has led to a demand for a discovery mechanism. To perform discovery, researchers have developed Matchmakers to match web service requesters and providers. Current Matchmakers do not support the matching of semantic web services that use different ontologies. Thus, even if the web service providers meet the requirement of web service requesters, such a match may be missed. This paper introduces a framework which supports matching web services using different ontologies. The Matchmaker algorithm, which incorporates a means of distinguishing different ontologies, is presented.
\end{abstract}

\section{Introduction}

Semantic web service is a technology which is based on semantic web and web service technologies. It has become a core technology in e-business due to its strengths in discovery, composition, invocation, monitoring and so on. Discovery is the most important task in the web because web services are useless if they cannot be found. To perform discovery, researchers have developed Matchmakers [1, 2, 3, 4] to match web service requesters with web service providers.

Current Matchmakers are adequate when the web service requester and provider use the same ontology. Unfortunately, they do not support the situation where a web service requester and provider use different ontologies. In the real world, a web service provider can provide an exact service to the requester even though both services use different ontologies. The web service requester and provider operate independently; therefore, they usually define their own ontologies to describe their services.

Hence, a Matchmaker that supports web services using different ontologies is extremely important and provides the motivation behind this work. In the proposed framework, we assume that the building and maintaining of the ontologies are outside the scope of our work. The rest of the paper is as follows. Section 2 describes the matchmaker algorithm. Related work in matching web services is presented in section 3 , followed by the conclusion and future work.

\section{Matching Algorithm}

\subsection{Semantic Web Service}

A semantic web service defined in languages such as DAML-S and OWL-S [5] includes four basic classes, namely Service, ServiceProfile, ServiceModel, and ServiceGrounding (figure 1). For matching, only ServiceProfile is used because it con- 


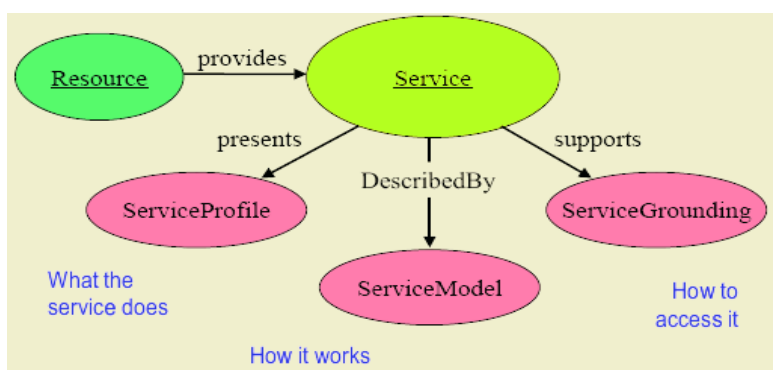

Fig. 1. Top level of the service ontology [5]

tains the description about the service to be discovered. The ServiceProfile [6] describes three basic types of information, namely, contact information, functional description of the service, and additional properties.

The contact information contains textDescription [6] which provides a brief description of the service. It describes what the service offers, or what service is requested. The functional description of the service is the most important declaration used for matching. It specifies the parameters of the inputs, outputs, preconditions, and effects of the service. The Profile also declares an operation of the web service.

\subsection{Matchmaker Description}

Before matching can take place, the web service providers must advertise their information to the Matchmaker and the advertised information will be stored in the Matchmaker. When a requester with a certain requirement wants to find a service, the Matchmaker will attempt to match the profiles of the requester with the advertised information of providers in its database. As the result of the matching, the Matchmaker will output a list of sufficiently similar [2] providers to the requested service. It is necessary to define the term "sufficiently similar". We define four degrees of similarity, namely, exact match, plug-in match, relaxed match, and fail match [2, 4]. Two web services are called sufficiently similar when the result of matching is Exact match, Plug-in match, or Subsumes match.

Exact match is the most accurate match. It happens when the two descriptions are semantically equivalent. In this case, two web services point to the same concept. Plug-in match is less accurate than exact match. This is when the advertisement service is more general than the requested service. Subsumes match is the least accurate. It is the inverse of Plug-in match and happens when the requested service is more general than the advertisement service. Fail match happens because of three reasons. Firstly, when there is no subsumption relation between advertisement and request. Secondly, when the requested service and advertisement service use different ontologies and two web services have different descriptions. Finally, it happens when the distance of two concepts in different ontologies exceeds a specific threshold (section 2.3).

\subsection{Matchmaker Algorithm}

The proposed matchmaker divides the matching into four stages [4] that include input matching, output matching, operation matching, and user-defined matching. Each 
stage is independent of the other. The algorithm uses [2] as a starting point, which can be described as follows:

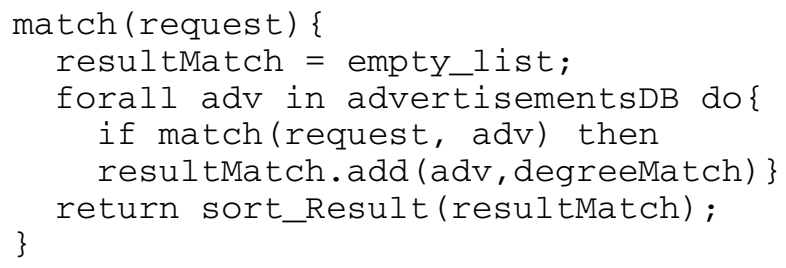

The requested service is matched against all the advertisements stored in the database of the matchmaker. If the advertisement service is sufficiently similar to the requested service, we add the advertised information and its degree of match to the resultMatch. After the requested service is matched with all advertisements, sort_Result function will return a sorted list with a number of advertisements which fits the requester, based on the degree of matching. The matching process is elaborated as follows:

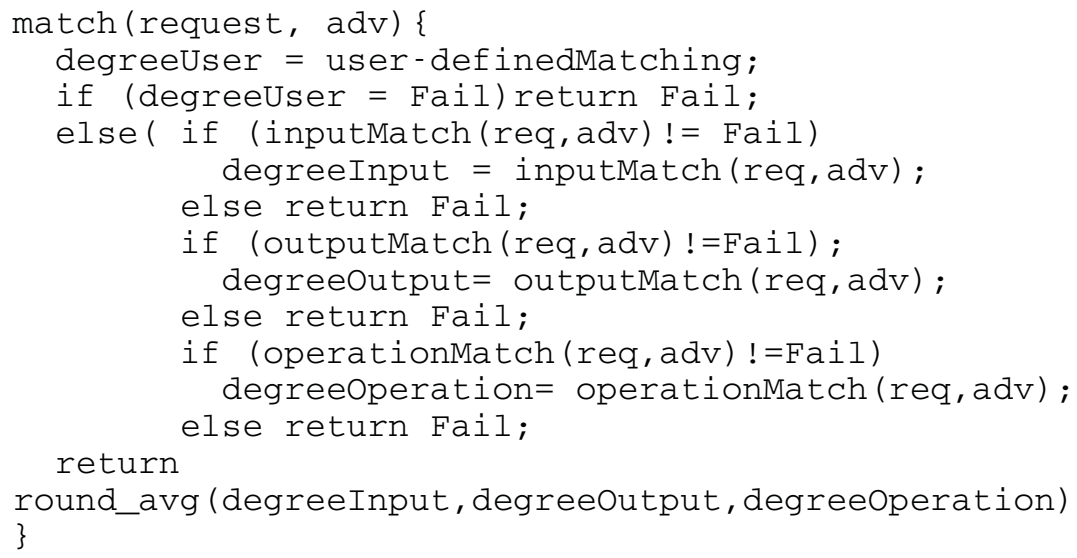

Users can refine the result by specifying the similarity degree of each stage. In addition, the user-defined stage can be used to narrow the result of matching by declaring some constraints or rules. For example, a requester wishing to buy a computer describes a web service with price as input and configuration of the computer as output. They may also declare more constraints relating to the computer. For instance, the computer must be manufactured by a particular company. These constraints or rules will be matched against the advertisement and the result of user-defined matching is "match" or "not match". If the advertisement does not match the constraints, the matching will fail. Otherwise, the final matching will be computed based on the average degree of input, output, operation matching.

Input matching first checks if two web services use the same ontology. If they do, we will match each input of the advertised service with an input of the request services. If they use different ontologies, we will check if the two services have the same description. If the descriptions are different, the two web services cannot be matched. In other words, matching fails. Otherwise, input matching computes the distance between two concepts. The algorithm for input matching is as follows: 


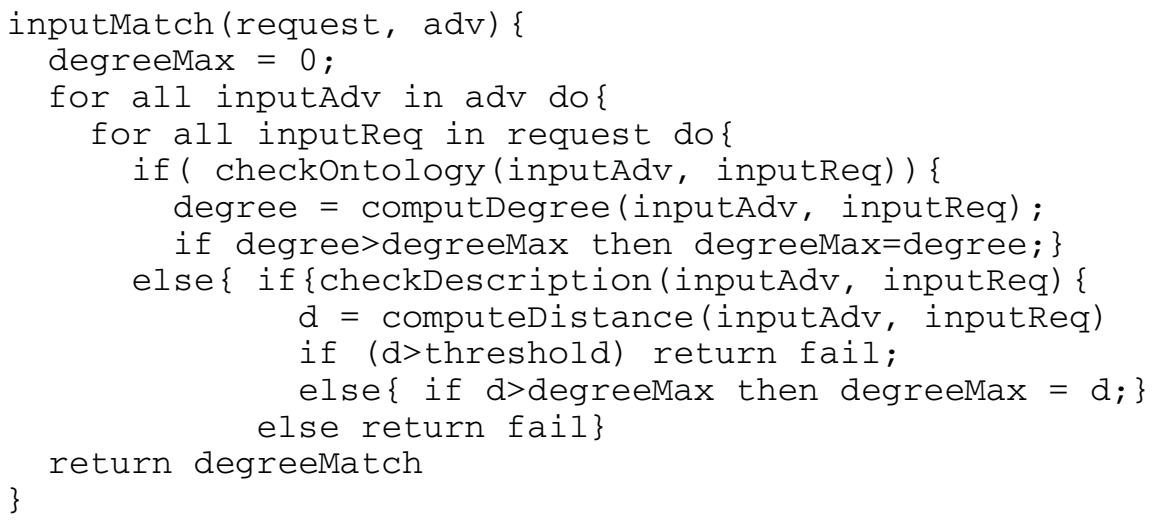

The threshold referred to in the algorithm is a value which depends on a specific application and domain. The input match will return the degree of the input. Both output and operation processing are carried out in the same manner as input. But in output matching, all output from the requested service will be matched with each output in the advertised service. In operation matching, service category of the requested and service category the advertised service are matched. The results of these two matching are the degree of output matching and operation matching respectively.

After matching two services, the semantic distance of the match will be the sum of all four stages. This will be stored in the database. After matching with all advertisements from the database, the matched list will be sorted by the degree of similarity of advertisement web service and returned to the requester.

The semantic distance within the same ontology is computed based on the relationship between two concepts in the ontology. We assume that A and B are two concepts of an ontology. The function to compute the distance between A and B is as follows:

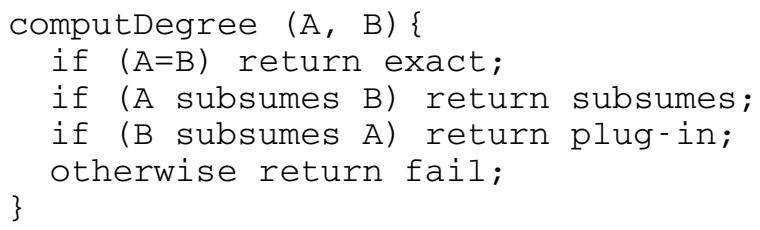

\subsection{Compute Distance and Description}

To check if two web services have the same description we use clustering technology. Clustering is a process that divides a set of items into groups of similar items [7]. Each group is a cluster and consists of items that are similar to each other. If two textDescription (section 2.1) belong to the same group, we say that they refer to the same function. Otherwise, two web services cannot be matched.

To compute the distance between two concepts from different ontologies, we use [8] to define rules to compute the semantic distance between labels, properties, superconcepts or sub-concepts of two concepts. The final distance of two concepts A and B is the summation of the three components with weight $w_{i}$.

$$
\operatorname{similarity}(A, B)=\sum_{i=1}^{n} w_{i} \operatorname{similarity}_{i}(A, B) \text {. }
$$


$w_{i}$ is application and domain specific. $n$ is number of rules. When requested and advertisement web service use different ontologies, similarity (A, B) will be used to compute the similarity matching of input, output, and operation matching.

\section{Related Work}

Since web services use different languages to describe the services and hence, are semantically incompatible, the LARKS (Language for Advertisement and Request for Knowledge Sharing) project [3, 4] defined a common language called ADCL (Agent Capability Description Language). LARKS includes five different filters for matching. However, ADCL is not a standard of W3C (www.w3c.org) and is difficult to write. Furthermore, LARKS does not support other commonly used languages such as DAML-S and OWL-S.

The Matchmaker from the collaboration between Toshiba and Carnegie Mellon University [10] is based on LARKS and the algorithm from [2] to overcome the drawbacks of LARKS. Similar to LARKS, it allows users to decide which filters they would like to use to narrow the result of matching. However, it has two drawbacks; first, it does not support operation matching which is very important in matching operations; second, the Text Filter which is useful to minimize missed matches is inefficient.

$\mathrm{Li}$ and Horrocks [1] have developed a framework for matchmaking based on DAML-S. It tries to match the profiles of requested service and advertised service respectively directly, instead of dividing the matching into several stages. Service profile is part of any web service description language and includes information such as input, output, precondition, effective, user information etc. Therefore, without dividing the matching into several stages, it will be difficult to automate reasoning techniques to compute semantic matching and the matching will be time-consuming.

The Matchmaker from TU-Berlin [4] is a good example of a matching algorithm. It is divided into four stages. By dividing the matching algorithm, the Matchmaker not only avoids the drawback from [1] but also helps users to choose the degree of matching from each stage. However, the matchmaker is quite simple and one of the drawbacks is that it is very difficult for users to utilize user-defined matching. With userdefined matching, the user must define their rules without user-friendly graphical user-interfaces.

All of the above matchmakers assume that the requested service and advertised services use the same ontology and support one semantic description language. The proposed Matchmaker supports matching of web services using different ontologies and different description languages.

\section{Conclusions and Future Work}

In this paper we have introduced an algorithm and engine which supports matching of web services using different ontologies. The proposed algorithm is based on $[2,4]$. The algorithm in [2] is extended to support matching of web services using different ontologies. It also supports the matching of web services which use different web service description languages such as OWL-S, DAML-S, and RDFS by using different reasoners. Each reasoner will be responsible for a different web services descrip- 
tion language. However, to determine if two web services have the same description by using clustering technology has limitations when the number of services is not large enough. The approach in computing the distance of two concepts from different ontologies is also too simplistic. Alternative solutions to these issues are being looked into, as well as implementation of the framework.

\section{References}

1. L. Li and I. Horrocks: A software framework for matching based on semantic web technology. $12^{\text {th }}$ International World Wide Web Conference (WWW 2003), pp. 331-339, Budapest, Hungary, 2003.

2. M. Paolucci, T. Kawamura, T. R. Payne, K. Sycara: Semantic Matching of Web services Capabilities. $1^{\text {st }}$ International Semantic Web Conference (ISWC 2002), pp. 333-347, Sardinia, Italy, 2002.

3. K. Sycara, S. Widoff, M. Klusch, J. Lu: LARKS: Dynamic Matchmaking Among Heterogeneous Software Agents in Cyberspace. Autonomous Agents and Multi- Agent Systems, Vol.5, pp.173-203, 2002.

4. M.C.Jaeger, S. Tang and C. Liebetruth: The TUB OWL-S Matcher. Available at http://ivs.tu-berlin.de/Projekte/owlsmatcher/index.html

5. W3C: OWL-S 1.0 Release. Available at: http://www.daml.org/services/owl-s/1.0/

6. OWL Profile: http://www.daml.org/services/owl-s/1.0/Profile.owl

7. P. Berkhin, Survey of Clustering Data Mining Techniques: Technical Report. Accure Software. Available at: http://citesseer.nj.nec.com/berkhin02servey.html

8. M. Ehrig, Y. Sure: Ontology Mapping - An Integrated Approach. $1^{\text {st }}$ European Semantic Web Symposium, Heraklion, Greece, 2004. 